\title{
Optimal Toll Locations and Levels in Congestion Pricing Schemes: a Case Study of Stockholm
}

Joakim Ekström, Leonid Engelson and Clas Rydergren

\section{Linköping University Post Print}

\section{Tweet}

N.B.: When citing this work, cite the original article.

This is an electronic version of an article published in:

Joakim Ekström, Leonid Engelson and Clas Rydergren, Optimal Toll Locations and Levels in Congestion Pricing Schemes: a Case Study of Stockholm, 2014, Transportation planning and technology (Print), (37), 4, 333-353.

Transportation planning and technology (Print) is available online at informaworldTM: http://dx.doi.org/10.1080/03081060.2014.897129

Copyright: Taylor \& Francis (Routledge): SSH Titles http://www.routledge.com/

Postprint available at: Linköping University Electronic Press http://urn.kb.se/resolve?urn=urn:nbn:se:liu:diva-76640 
Optimal Toll Locations and Levels in Congestion Pricing Schemes: a Case Study of Stockholm

Joakim Ekström $^{\mathrm{a}^{*}}$, Leonid Engelson ${ }^{\mathrm{b}}$ and Clas Rydergren ${ }^{\mathrm{a}}$

${ }^{a}$ Department of Science and Technology, Linköping University, Norrköping, Sweden

*Corresponding author. Email: joakim.ekstrom@liu.se 


\section{Optimal Toll Locations and Levels in Congestion Pricing Schemes: a Case Study of Stockholm}

As congestion pricing has moved from theoretical ideas in the literature to real world implementations, the need for decision support when designing the pricing schemes has become evident. This paper deals with the problem of finding optimal toll levels and locations in a road traffic network, and presents a case study of Stockholm. The optimization problem of finding optimal toll levels, given a predetermined cordon, and the problem of finding both optimal toll locations and levels are presented, and previously developed heuristics are used for solving these problems. For the Stockholm case study, the possible welfare gains of optimizing the toll levels in the current cordon, and optimizing both the toll locations and their corresponding toll levels are evaluated. It is shown that by tuning the toll levels in the current congestion pricing cordon used in Stockholm, the welfare gain can be significantly increased, and furthermore improved by allowing a toll on the bypass highway "Essingeleden". It is also shown that by optimizing both the toll locations and levels, a congestion pricing scheme with welfare gain close to what can be achieved by marginal social cost pricing, can be designed with tolls being located on only a fourth of the tollable links.

Keywords: congestion pricing; road tolls; bilevel optimization; user equilibrium, network design,

\section{Introduction}

Without any intervention from the road authority, the users of a transportation network are assumed to make travel decisions that minimize their individual travel cost. This may lead to an inefficient usage of the transportation facilities, which is experienced as increased congestion in everyday traffic, in metropolitan areas across the world. A more efficient usage can, however, be achieved if the users make travel choices with respect to the effect that these choices have on the other users' travel cost. One way of achieving such decisions is to introduce a congestion pricing scheme, which has recently, in Sweden, been done in Stockholm and Gothenburg. 
As congestion pricing has moved from an idea in transportation literature to practical implementations, the need for decision support when designing the pricing schemes has become evident. In this paper, previously developed methods (Ekström 2008; Ekström, Engelson and Rydergren 2009), based on mathematical models with the objective to maximize the social surplus, for designing link-based congestion pricing schemes, are used in a case study of designing congestion pricing schemes in Stockholm. The study includes tuning of the current toll levels at existing toll locations, extending the existing cordon with additional toll locations, and a completely new design of the congestion pricing scheme. It is, however, important to recognize that the mathematical model used in this paper is limited to what can be measured and modelled. When introducing a congestion pricing scheme in practice there will be practical and political considerations that also have to be taken into account in the final design, considerations which cannot be included in a mathematical model.

Generally, the most efficient usage of a scarce resource is accomplished when the users' cost is equal to the social cost. Therefore, the optimal usage of a road network is achieved when the tolls are equivalent to the marginal impact that one additional user has on the other users' total travel cost. This pricing principle is often referred to as marginal social cost pricing (MSCP) (Beckmann, McGuire and Winsten 1956) and was first discussed by Pigou (1920), followed by Knight (1924). The idea of MSCP in a traffic network is theoretically appealing, but in practice not very likely to be implemented. MSCP requires the collection of tolls on every road segment with congestion, and does not take the setup and operational costs of the toll collection system into account. Therefore, there is a need for alternative and more practical congestion pricing schemes. When restrictions are imposed on the congestion pricing scheme design, the optimal design is usually said to be second-best (Verhoef, Nijkamp 
and Rietveld 1996) as opposed to first-best when there are no restrictions.

The link flow and travel demand pattern in the traffic network, resulting from MSCP tolling, is usually referred to as system optimal (SO). The MSCP tolling scheme is, however, not the only pricing scheme which achieve SO flows and demands, and in Yildirim and Hearn (2005) a method for finding other pricing schemes which achieve SO flows and demands is presented. For the case when the demand is elastic Larsson and Patriksson (1998) show that the total toll revenue is unique for all pricing schemes leading to SO link flow and travel demand (later also shown in Yin and Lawphongpanich [2009]). Larsson and Patriksson (1998) further conclude that even though the collection of MSCP tolls can be transferred between different links on a route, it is only possible in practice when several links can be replaced by a single one in the SO solution. Thus the discussion in Yildirim and Hearn (2005), related to finding the congestion pricing scheme with the minimum number of located tolls which still gives SO link flows and travel demands, is of limited practical interest.

To set up and operate a congestion pricing scheme can, even with the advanced technologies available today, be a costly task. Thus there is an interest in finding a system which produces a high benefit at a low cost. The objective is to maximize the social surplus $(S S)$, which is the user surplus $(U S)$ plus the operator surplus $(O S)$. The user surplus is the user benefits $(U B)$ (given by the Marshallian measure [Zerbe and Dively 1994]) minus the total user costs (UC) (travel time and paid tolls), and the operator surplus is the total toll revenues $(R)$, sometime referred to as the operator benefit (Verhoef 2002), minus the cost of collecting the tolls $(C)$, which includes setup and operational costs of the toll collection system. The social surplus can thus be expressed as $S S=U S+O S=U B-U C+R-C$. 
In this paper the road users are assumed to be distributed according to a user equilibrium (Wardrop 1952). To maximize the social surplus can then be formulated as a bilevel optimization problem (for an overview of bilevel optimization problems see Luo, Pang and Ralph [1996]). On the upper level the social surplus is optimized by adjusting the decision variables, which are the toll locations and levels. On the lower level, the user equilibrium link flows and travel demands are computed for fixed values on the decision variables, by solving a convex program (Sheffi 1985), with link flows and travel demands being the traffic state variables. This implicit relationship between decision and traffic state variables makes the bilevel optimization problem both nonconvex and non-smooth, and thus difficult to solve for a global optimal solution (for an overview of bilevel optimization problems in a traffic planning context see e.g. Migdalas [1995]). To solve the bilevel problem, which will be denoted as the toll design problem (TDP), different (meta-) heuristics have previously been suggested. For instance Shepherd and Sumalee (2004) developed a genetic algorithm which is extended in Sumalee (2004) to find closed cordon schemes. In Zhang and Yang (2004) the problem of finding closed cordon structures which maximize the social surplus is addressed by a combination of genetic algorithms and simulated annealing. A closely related problem is the problem of finding toll levels when the toll locations are predetermined. This is still a non-convex and non-smooth optimization problem, and will be referred to as the toll level problem (TLP). Note that for the TLP, the operator cost will be constant and is therefore sometimes excluded from the optimization problem. In Verhoef, Nijkamp and Rietveld (1996) and Liu and McDonald (1999) properties of the TLP are discussed, and in Verhoef (2002), Lawphongpanich and Hearn (2004) and Shepherd and Sumalee (2004) methods for finding optimal toll levels are 
developed.

The congestion pricing trial in Stockholm 2006, and the permanent congestion pricing scheme, started in 2007, has been studied in several papers. An overview of the effects during the trial period is presented in Eliasson et al. (2009) and the behavioural adjustments and equity effects are studied in Karlström and Franklin (2009). A costbenefit analysis of the current congestion charging system is given in Eliasson (2009). These studies are based on measured traffic data and travel surveys, and not on model estimations. Before the trial in Stockholm, several studies of the effects of different designs of the congestion pricing scheme were carried out (Transek, 2003, 2004) using the Swedish national travel demand forecasting tool Sampers (described in e.g. Beser and Algers [2002]), which include a detailed network model of the roads in the Stockholm region. Besides Sampers, the same network model of Stockholm has been used together with the regional demand forecasting models T/RIM (presented in e.g. Engelson and Svalgård [1995]) and SIMS (described in e.g. Algers et al. [1996]), for estimating the effects of different designs of the Stockholm congestion pricing scheme (for a comparison of the different demand forecasting models see Transek [2003]). In our case study, a more aggregated road network is used, including only the main roads in Stockholm, and the demand model (based on data from T/RIM) merely includes the choice between car and public transport, given a fixed total demand. The purpose of the case study presented in this paper is to give some insight on optimal design of congestion pricing schemes in Stockholm, but also to show the applicability of the sensitivity analysis based method presented in Ekström (2008) and of the smoothening technique presented in Ekström, Engelson and Rydergren (2009). The sensitivity analysis based heuristic is used for tuning the toll levels in the current congestion pricing scheme in Stockholm, in order to maximize the social surplus, and the 
smoothening technique is used for maximizing the social surplus, by adjusting both the toll locations and their corresponding toll levels.

The remaining part of this paper is outlined as follows. In Section 2 the modelling framework and solution algorithms are presented. The Stockholm case study is presented in Section 3, and in Section 4 we conclude the results.

\section{Methodology}

\subsection{The road traffic model}

The traffic network is modelled by a set of links $A$ and a set of origin destination (OD) pairs $I$. For each link $a \in A$ there is a travel cost function $c_{a}$ of car flow $v_{a}$ and link toll $\tau_{a}$ :

$$
c_{a}\left(\tau_{a}, v_{a}\right)=\alpha t_{a}\left(v_{a}\right)+\frac{\tau_{a}}{\beta},
$$

where $t_{a}\left(v_{a}\right)$ is the link travel time function, $\alpha$ the mean value of time (VOT) which transforms time into a monetary cost, and $\beta$ the car occupancy which give the mean number of persons in each car. Note that $c_{a}\left(v_{a}\right)$ is the cost perceived by one traveller, not one car, and since the toll, $\tau_{a}$, is charged for each car it is divided by $\beta$ in (1), in contrast to $t_{a}\left(v_{a}\right)$ which is the travel time perceived by each traveller in the same car. For the Stockholm case study presented in this paper the link travel time functions takes on the form

$$
t_{a}\left(v_{a}\right)=L_{a}\left(\frac{v_{a}}{p_{1, a} n_{a}}+p_{2, a}\left(1+\left(\frac{v_{a}}{p_{3, a} n_{a}}\right)^{p_{4, a}}\right)\right),
$$


where $L_{a}$ is the link length, $n_{a}$ the number of lanes, and $p_{1, a}-p_{4, a}$ link type specific parameters. The road capacity for link $a$ is given by $p_{3, a} n_{a}$.

For each OD pair $i \in I$ there is a set of routes $\Pi_{i}$, with $f_{p}$ travellers on each route $p \in \Pi_{i}$. The car flow, $v_{a}$, on link $a$ is given by

$$
v_{a}=\frac{1}{\beta} \sum_{i \in I} \sum_{p \in \Pi_{i}} f_{p} \delta_{p}^{a}
$$

where $\delta_{p}^{a}$ takes the value of 1 if route $p$ traverses link $a$, and 0 otherwise.

The road users are assumed to be distributed according to Wardrop's user equilibrium (UE) (Wardrop, 1952), which assumes that the road users have perfect information of travel costs in the network and make decisions which maximize their individual utility. The demand is assumed to be elastic, and given for OD pair $i \in I$ by the decreasing demand function $q_{i}=D_{i}\left(\pi_{i}\right)$, with its inverse formulation $\pi_{i}=D_{i}^{-1}\left(q_{i}\right)$, where $\pi_{i}$ is the minimum travel cost (in monetary terms) and $q_{i}$ the travel demand (in the unit of travellers per hour) in OD pair $i \in I$. For the Stockholm case study the elastic demand function will be given by the pivot point version of the binomial logit model (Kumar, 1980). For each OD pair $i \in I$, the car travel demand is calculated as

$$
q_{i}=D_{i}\left(\pi_{i}\right)=T_{i} \frac{A_{i}}{A_{i}+K_{i} e^{\lambda\left(\pi_{i}-\pi_{i}^{0}\right)}}
$$

where $\pi_{i}\left(\pi_{i}^{0}\right)$ is the minimum travel cost by car in the tolled (non-tolled) scenario respectively, and the parameters $A_{i}$ and $K_{i}$ are the number of travellers by car and by public transportation in the non-tolled scenario (corresponding to the car travel $\left.\operatorname{cost} \pi_{i}^{0}\right) . T_{i}$ is the total demand in OD pair $i$, with $T_{i}=A_{i}+K_{i}$, and $\lambda>0$ is the dispersion parameter. In the pivot point version of the binomial logit model the travel time for public transport is assumed to be fixed, and not depending on congestion on the 
road or in the public transportation system itself. Note that the number of car users in OD pair $i$ is always less than $T_{i}$ and when $\pi_{i}$ increases the number of travellers by car will decrease and the number of travellers by public transport increase.

The user equilibrium with elastic demand, given a toll vector $\bar{\tau}$, can be formulated as the complementarity problem

$$
\begin{array}{ll}
f_{p}>0 \Rightarrow \sum_{a \in A} c_{a}\left(\bar{\tau}_{a}, v_{a}\right) \delta_{p}^{a}=\pi_{i}, & p \in \Pi_{i}, i \in I \\
f_{p}=0 \Rightarrow \sum_{a \in A} c_{a}\left(\bar{\tau}_{a}, v_{a}\right) \delta_{p}^{a} \geq \pi_{i}, & p \in \Pi_{i}, i \in I \\
q_{i}>0 \Rightarrow \pi_{i}=D_{i}^{-1}\left(q_{i}\right), & i \in I \\
q_{i}=0 \Rightarrow \pi_{i} \geq D_{i}^{-1}\left(q_{i}\right), & i \in I,
\end{array}
$$

which states that any used route in OD pair $i$ must have a travel cost equal to the minimum travel cost in OD pair $i$. An equivalent convex program can be formulated as (see e.g. Sheffi [1985] and Patriksson [1994])

$$
\min _{q, v} G(\bar{\tau}, q, v)=\beta \sum_{a \in A} \int_{0}^{v_{a}} c_{a}(\bar{\tau}, x) d x-\sum_{i \in I} \int_{0}^{q_{i}} D_{i}^{-1}(w) d w
$$

subject to

$$
\begin{array}{cl}
\sum_{p \in \Pi_{i}} f_{p}=q_{i}, & i \in I \\
f_{p} \geq 0, & p \in \Pi_{i}, i \in I \\
q_{i} \geq 0, & i \in I \\
v_{a}=\frac{1}{\beta} \sum_{i \in I} \sum_{p \in \Pi_{i}} f_{p} \delta_{p}^{a}, & a \in A .
\end{array}
$$


When the relationship between travel cost and travel demand is given by the pivot point version of the binomial logit model, we can rewrite (4) as (Ekström, 2008)

$$
\begin{aligned}
& \min _{q, v} G(\bar{\tau}, q, v)=\beta \sum_{a \in A} \int_{0}^{v_{a}} c_{a}(\bar{\tau}, x) d x-\sum_{i \in I}\left[\pi_{i}^{0} q_{i}+\frac{q_{i}}{\lambda} \ln \frac{A_{i}\left(T_{i}-q_{i}\right)}{K_{i} q_{i}}+\frac{T_{i}}{\lambda} \ln \frac{T_{i}}{T_{i}-q_{i}}\right] \\
& \text { subject to } \\
& \sum_{p \in \Pi_{i}} f_{p}=q_{i}, \quad i \in I \\
& f_{p} \geq 0, \quad p \in \Pi_{i}, i \in I \\
& q_{i} \geq 0, \quad \quad i \in I \\
& v_{a}=\frac{1}{\beta} \sum_{i \in I} \sum_{p \in \Pi_{i}} f_{p} \delta_{p}^{a},
\end{aligned}
$$

which can be solved by the partial linearization method presented in Evans (1976), in which a fixed demand UE problem is solved repeatedly and the demand is updated in between. For solving the fixed demand UE problem, the Disaggregated Simplicial Decomposition (DSD) algorithm (Larsson and Patriksson, 1992) will be used. The DSD algorithm can be restarted from a previous solution, with the demand data updated, and re-optimize the previous solution efficiently. This is a useful feature when solving the fixed demand UE problems in Evans partial linearization method.

\subsection{The toll level problem}

In the toll level problem (TLP) the objective is to maximize the social surplus and the decision variables are the toll levels for each toll location. The toll locations are given exogenously and the set of tolled links $(T)$ is given by

$$
T:\left\{\tau_{a} \mid \tau_{a} \leq g_{a} M\right\}
$$

where $M$ is a large positive number, and $g_{a}$ is a parameter which takes on the value of 1 if link $a$ is tollable and 0 otherwise.

The social surplus measure $(S S)$ consists of the user surplus (US), 


$$
U S(\tau, q(\tau), v(\tau))=\sum_{i \in I} \int_{0}^{q_{i}(\tau)} D_{i}^{-1}(w) d w-\beta \sum_{a \in A}\left(\alpha t_{a}\left(v_{a}(\tau)\right)+\frac{\tau_{a}}{\beta}\right) v_{a}(\tau)
$$

in which the first sum is the user benefits (given by the Marshallian measure [Zerbe and Dively, 1994]) and the second sum the user costs, plus the operator surplus (OS)

$$
O S(\tau, q(\tau), v(\tau))=\sum_{a \in A}\left(\tau_{a} v_{a}(\tau)-C_{a} g_{a}\right)
$$

which is the collected tolls minus the cost of collecting the tolls. $q(\tau)$ and $v(\tau)$ are given by the solution to the user equilibrium problem, either in the form of (4) or (5), and $C_{a}$ equals the cost of setting-up and operating a toll on link $a$.

Let $S S_{0}$ be the social surplus associated with the zero toll vector, and let $S S(\tau, q(\tau), v(\tau))$ be the social surplus associated with toll vector $\tau$. The objective to maximize can then be formulated as

$$
\max _{\tau \in T} F_{\mathrm{TLP}}(\tau)=S S(\tau, q(\tau), v(\tau))-S S_{0}
$$

Note that $S S_{0}$ is constant, and with (7) and (8) inserted, the objective can be expressed as

$$
\begin{aligned}
\max _{\tau \in T} & F_{\mathrm{TLP}}(\tau, q(\tau), v(\tau))=U S(\tau, q(\tau), v(\tau))+O S(\tau, q(\tau), v(\tau)) \\
& =\sum_{i \in I} \int_{0}^{q_{i}(\tau)} D_{i}^{-1}(w) d w-\beta \sum_{a \in A}\left(\alpha t_{a}\left(v_{a}(\tau)\right)+\frac{\tau_{a}}{\beta}\right) v_{a}(\tau)+\sum_{a \in A}\left(\tau_{a} v_{a}(\tau)-C_{a} g_{a}\right)-S S_{0} \\
& =\sum_{i \in I} \int_{0}^{q_{i}(\tau)} D_{i}^{-1}(w) d w-\beta \sum_{a \in A} \alpha t_{a}\left(v_{a}(\tau)\right) v_{a}(\tau)-\sum_{a \in A} C_{a} g_{a}-S S_{0} .
\end{aligned}
$$

Inserting the demand function (3) in (9), the optimization program can be expressed as (for details see Ekström [2008])

$$
\max _{\tau \in T} F_{\mathrm{TLP}}(v(\tau), \pi(\tau), \tau)=\frac{1}{\lambda} \sum_{i \in I} T_{i} \ln \frac{A_{i} e^{\lambda\left(\pi_{i}^{0}-\pi_{i}(\tau)\right)}+K_{i}}{T_{i}}+\sum_{a \in A}\left(\tau_{a} v_{a}(\tau)-C_{a} g_{a}\right)
$$

where $\pi_{i}$ is the minimum OD travel cost in OD pair $i$ in $I$, and can easily be extracted from the solution to (5). The TLP, either in the form of (9) or (10) is a bilevel program 
which in the general case is non-convex. Even though it is possible to transform the problem into a single level mathematical program with equilibrium constraints (MPEC) (Lawphongpanich and Hearn 2004), the non-convex nature is not changed (Luo, Pang and Ralph 1996). Also, $F_{\text {TLP }}$ is non-differentiable at solutions for which the set of used routes (in the user equilibrium problem) with minimal travel cost is changed.

To solve the TLP different methods have previously been suggested. Derivative based methods are presented in Yang and Lam (1996), Verhoef (2002), (Lawphongpanich and Hearn 2004) and Ekström (2008), and in Shepherd and Sumalee (2004) and Yang and Zhang (2003) stochastic search methods are applied.

In Patriksson and Rockafellar (2003) a framework for performing sensitivity analysis of traffic equilibria with elastic demand is presented. The results from Patriksson and Rockafellar have been applied to obtain gradients (or subgradients) in various bilevel programs, in which the lower level program represents a user equilibrium problem; in Josefsson and Patriksson (2007) for a network design problem, in Ekström (2008) and Ekström, Engelson and Rydergren (2009) for toll level and location problems, and in Lundgren and Peterson (2008) and Lederman and Wynter (2011) for OD estimation problems. In this paper the ascent method presented in Ekström (2008) and Ekström, Engelson and Rydergren (2009) will be used to solve the TLP.

By performing sensitivity analysis on the current equilibrium link flows, OD flows, and OD travel costs $v^{*}, q^{*}$ and $\pi^{*}$, at the toll level $\bar{\tau}$, the subgradients $\partial v_{a}(\bar{\tau}), a \in A$, and $\partial q_{i}(\bar{\tau}), i \in I$, can be computed (Josefsson and Patriksson 2007) by solving one sensitivity analysis problem for each tollable link. Also, the subgradient $\partial \pi(\bar{\tau}), i \in I$ can be computed for the case when the demand function is given by (3) (for details see Ekström [2008]). The sensitivity analysis problem is essentially a user 
equilibrium problem with linear link cost and inverse demand functions. The link cost function for link $a$ is replaced by $c_{a}^{\prime}\left(v_{a}^{\prime}, \tau_{a}^{\prime}\right)=\frac{\tau_{a}^{\prime}}{\beta}+\frac{\partial c_{a}\left(v_{a}^{*}, \bar{\tau}_{a}\right)}{\partial v_{a}} v_{a}^{\prime}$ and the inverse demand function for OD pair $i$ is replaced by $D_{i}^{-1^{\prime}}\left(q_{i}^{\prime}\right)=\frac{\partial D_{i}^{-1}\left(q_{i}^{*}\right)}{\partial q_{i}} q_{i}^{\prime}$, where $v^{\prime}$ and $q^{\prime}$ can be interpreted as vectors of directional derivatives in the directional change of link toll levels, given by the vector $\tau^{\prime}$. Setting $\tau_{a}^{\prime}=1$ for link $a=b$ and $\tau_{a}^{\prime}=0$ for any other links $a \neq b$ will give the link flow and demand perturbations, $v^{\prime}$ and $q^{\prime}$, which correspond to a positive unit change of the toll level on link $b$. By solving the sensitivity analysis problem for each link $b \in A$, with $\tau_{b}^{\prime}=1$ and $\tau_{a}^{\prime}=0$ for every link $a \neq b$, the subgradients $\partial v_{a}(\bar{\tau})$ and $\partial q_{i}(\bar{\tau})$ can be constructed.

If $F_{\mathrm{TLP}}(v(\tau), \pi(\tau), \tau)$ is differentiable with respect to $\tau$ in the point $\bar{\tau}$, $\nabla v_{a}=\partial v_{a}, \nabla q_{i}=\partial q_{i}$ and $\nabla \pi_{i}=\partial q_{i}$, if $F_{\mathrm{TLP}}(v(\tau), \pi(\tau), \tau)$ is non-differentiable in $\bar{\tau}$ then the subgradients will be used to approximate the gradients. By the rule of chain $\nabla F_{\mathrm{TLP}}$ can then be computed, and used in an ascend method. Since there can exist cases when $F_{\mathrm{TLP}}(v(\tau), \pi(\tau), \tau)$ is non-differentiable it is not guaranteed that $\nabla F_{\mathrm{TLP}}$ will always give an ascent direction, and the line search (in the ascent method) is therefore used with a minimum step length in Ekström (2008).

Since the sensitivity analysis problem essentially is a user equilibrium problem Josefsson and Patriksson (2007) discuss how to extend the DSD algorithm for solving the sensitivity analysis problem, for the case when the demand is fixed. For more details on the sensitivity analysis problem we refer to Patriksson and Rockafellar (2003) and Josefsson and Patriksson (2007), and for details on the sensitivity analysis based ascent 
method used for solving the TLP (either on the form (9) or (10) we refer to Ekström (2008).

\subsection{The toll design problem}

In the toll design problem (TDP) the objective is to find both the toll locations and their corresponding levels, which maximize the social surplus. The set of tollable links is still given by (6), but the cost associated with each toll location is now given by $C_{a} \operatorname{sign}\left(\tau_{a}\right)$, where the function sign of real argument $x \geq 0$ is defined as

$$
\operatorname{sign}(x)= \begin{cases}1, \text { if } & x>0 \\ 0, \text { if } & x=0 .\end{cases}
$$

By using the sign-function, there will only be a cost associated with the toll location if the toll level is positive, and the toll locations are given implicitly by the positive toll levels. With the demand given by (3), the toll design problem is formulated as

$$
\max _{\tau \in T} F_{\mathrm{TDP}}(v(\tau), \pi(\tau), \tau)=\frac{1}{\lambda} \sum_{i \in I} T_{i} \ln \frac{A_{i} e^{\lambda\left(\pi_{i}^{0}-\pi_{i}(\tau)\right)}+K_{i}}{T_{i}}+\sum_{a \in A}\left(v_{a}(\tau) \tau_{a}-C_{a} \operatorname{sign}\left(\tau_{a}\right)\right)
$$

To solve this problem a heuristic procedure (referred to as the approximation approach in Ekström, Engelson and Rydergren (2009) is applied, which combines a smoothing technique of the objective function with the sensitivity analysis based algorithm described in the previous section. The general idea of the smoothing technique is to replace the sign function in (11), with a continuous function. Note that due to the bilevel nature of the problem, the objective function may still be non-smooth with respect to the toll levels.

Comparing the TLP (10) with the TDP (11), the sign function in (11) gives the problem a combinatorial structure which makes derivative based solution methods 
unsuitable. In the heuristic approach we will smoothen the discrete part of the objective function by approximating the sign function with the continuous function

$$
\gamma\left(\tau_{a}, k\right)=\frac{2}{1+e^{-k \tau_{a}}}-1
$$

where $k$ determines the accuracy of the approximation. Note that $\gamma\left(\tau_{a}, k\right)=0$ at $k=0$, and for any $\tau_{a} \geq 0, \gamma\left(\tau_{a}, k\right)$ tends to $\operatorname{sign}\left(\tau_{a}\right)$ when $k$ tends to infinity.

Replacing $\operatorname{sign}\left(\tau_{a}\right)$ in problem $(11)$ by $\gamma\left(\tau_{a}, k\right)$ gives

$$
\max _{\tau \in T} F_{\mathrm{TDP}-\mathrm{S}}(v(\tau), \pi(\tau), \tau)=\frac{1}{\lambda} \sum_{i \in I} T_{i} \ln \frac{A_{i} e^{\lambda\left(\pi_{i}^{0}-\pi_{i}(\tau)\right)}+K_{i}}{T_{i}}+\sum_{a \in A}\left(v_{a}(\tau) \tau_{a}-C_{a} \gamma_{a}\left(\tau_{a}, k\right)\right),
$$

which is solved by the same sensitivity analysis based ascent method as the TLP. Starting with a low value of $k$, problem (12) is solved to a local optimum. The parameter $k$ is then increased and (12) is re-solved with the previous solution as the new starting solution. The iterations are repeated until the difference between the objective function values in (11) and (12) is small, and as $k$ is increased $F_{\text {TDP-S }}$ asymptotically converges to $F_{\mathrm{TDP}}$. Note that the information transferred between the iterations is the toll levels, and the toll locations are given implicitly by the toll levels. When the accuracy of the approximation is gradually increased, it is, however, less likely that the toll locations are changed, and in the final iteration it is in practice a TLP that is solved. Note that setting $k=0$ in (12) results in a TLP with the fixed set of tollable links given by $T$, and if all links are tollable the link flow and demand will correspond to the SO solution. Solving problem (10), with the sensitivity analysis based ascent method, will in each iteration require at least one user equilibrium problem to be solved and also one sensitivity analysis problem to be solved for each tollable link. Note that solving problem (11), with the smoothening technique require a series of approximation problems (12) to be solved. Thus the computational burden of solving 
(10) and (11) will depend on the network size (number of links and OD pairs), the level of congestion, and the number of tollable links. For a more detailed description of the method, and the complete algorithm, see Ekström (2008).

\section{Case study}

\subsection{The Stockholm model}

The Stockholm network has 392 links (312 if the connectors to the origin/destination zones are not counted) and 40 zones which give 1560 OD pairs, and will model the car traffic in the Stockholm region during the morning rush hour. To reduce the computational time, one can identify nodes in the network which only connect two links (only counting one direction), and locate a toll to either one of these, but not both. For the Stockholm network, this reduces the number of tollable links from 312 to 291.

In this study, an aggregated traffic network of the Stockholm region has been used (Figure 1), with travel time functions on the form (2) and travel demand functions on the form (3). The total demand of car and public transport (for travellers with access to car) during the morning rush hour, for each OD-pair, is based on data from the demand forecast model T/RIM. The T/RIM data is, however, given for a full Stockholm network with about 1100 links and 1250 zones. The zones in the full network have thus been aggregated to the zones in the smaller aggregated network used in this study. The dispersion parameter $\lambda=0.07$ and the car occupancy $\beta=1.13$ travellers per car are also provided from the T/RIM model. It is possible that the aggregation of the travel demand model and its usage with the aggregated traffic network, without further calibrations, will result in higher link flows compared with results from other models. In Table 1, link flows produced by the aggregated model for the no toll solution is compared to results presented in Transek (2003) from using Sampers, SIMS and T/RIM 
on the full size Stockholm network. The total flows are compared for the bypass highway "Essingeleden" and for the crossing of the current toll cordon. For "Essingeleden" the difference between all four models are small, but for the crossing of the current toll cordon the total flow range between 38357 and 47922 vehicles per hour, and the total flow produced by the aggregated model is very close to the flow from the T/RIM model. It should be noted that all four models assign flow close to the model capacity on "Essingeleden". For Sampers, SIMS and T/RIM the same network layout (the full network model) and travel cost functions are used, and for the aggregated model the travel cost functions for "Essingeleden" are similar to what is used in the full network model. Thus the variation in link flows can be expected to be small for "Essingeleden", between the different models. The aggregated model and the Sampers model are further compared in Table 2, both for the flows with the no toll solution, and for the predicted effect of the current congestion pricing cordon in Stockholm. Even though the total flow on "Essingeleden" is similar for the two models, the aggregated model gives higher flow for the northbound traffic and lower flow for the southbound traffic. The northbound flow on "Essingeleden" from the aggregated model is close to the model capacity in the non-tolled case, and while Sampers give an increase of $8 \%$ with the current congestion pricing scheme in Stockholm, the flow is only increased by $1 \%$ with the aggregated model. Also, the reduction in outbound flow across the current congestion pricing cordon seems to be high compared with the Sampers model. 


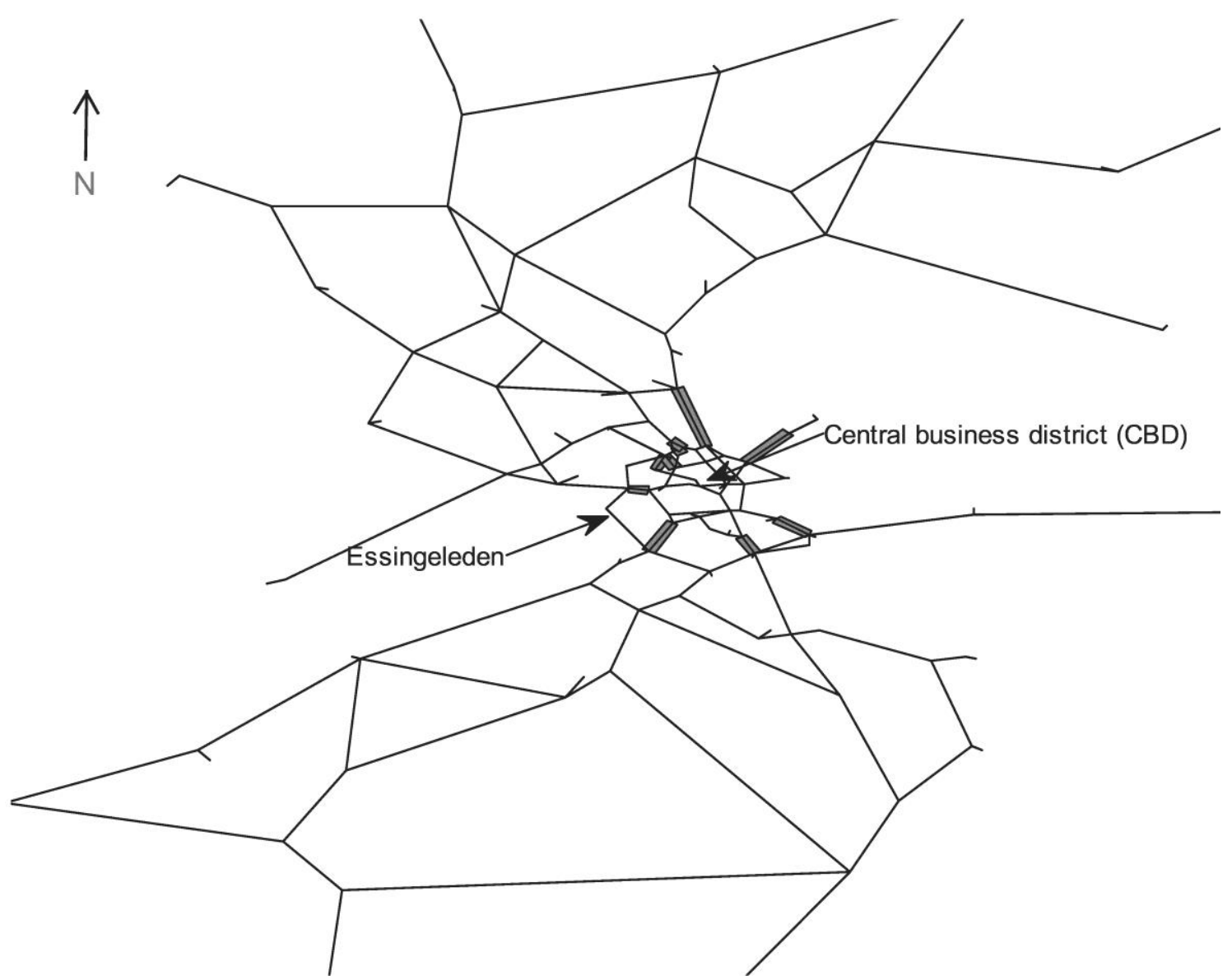

Figure 1: The Stockholm network. Tolled links in the currently implemented cordon in Stockholm are marked by grey bars.

The unit of account for costs and benefits in the objective function can either be consumer prices or producer prices. The users' benefits and costs of travelling are usually given in consumer prices to reflect that the users relate their costs and benefits of travelling to consumption of alternative goods with prices including value added tax. The operators investment costs are, however, usually given in producer prices to reflect that the operator is not charged value added tax on investment costs. To convert from producer prices to consumer prices a factor equal to 1.21 , which represents the average indirect tax on consumer goods in Sweden, is therefore used (SIKA 2009). In this case study all costs and benefits are given in Swedish currency (SEK) and in consumer prices, with the value of time set to 1.2 SEK per minute. The collection cost (in 
consumer prices) is given per peak hour, and is for each link computed as $C_{a}=500+500 n_{a}$, where $n_{a}$ is the number of lanes for link $a$. The mean collection cost is 1436 SEK per peak hour and toll location (1 189 SEK in producer prices).

Table 1: Traffic flow predictions, in vehicles per hour, computed with different models for the morning rush hour. Source for Sampers, SIMS and T/RIM: Transek (2003).

\begin{tabular}{ccc}
\hline Model & $\begin{array}{c}\text { Essingeleden } \\
\text { all directions }\end{array}$ & $\begin{array}{c}\text { Across the current cordon } \\
\text { all directions }\end{array}$ \\
\hline Sampers & 11033 & 38357 \\
SIMS & 11639 & 47922 \\
T/RIM & 11137 & 41244 \\
Aggregated model & 11356 & 41731 \\
\hline
\end{tabular}

Table 2: Modelled traffic flows for Stockholm, with and without the current congestion pricing cordon in Stockholm. Source for Sampers: Transek (2004).

\begin{tabular}{|c|c|c|c|c|}
\hline & \multicolumn{2}{|c|}{ Sampers } & \multicolumn{2}{|c|}{ Aggregated model } \\
\hline & Without (vec/h) & $\begin{array}{l}\text { With (change } \\
\text { of flow in \%) }\end{array}$ & Without (vec/h) & $\begin{array}{l}\text { With (change } \\
\text { offlow in \%) }\end{array}$ \\
\hline $\begin{array}{l}\text { Essingeleden } \\
\text { Both directions }\end{array}$ & 11639 & +10 & 11356 & +6 \\
\hline $\begin{array}{l}\text { Essingeleden } \\
\text { Northbound }\end{array}$ & 6032 & +8 & 6919 & +1 \\
\hline $\begin{array}{l}\text { Essingeleden } \\
\text { Soutbound }\end{array}$ & 5001 & +12 & 4437 & +13 \\
\hline $\begin{array}{l}\text { Across cordon } \\
\text { Both directions }\end{array}$ & 38357 & -33 & 41731 & -37 \\
\hline $\begin{array}{l}\text { Across cordon } \\
\text { Inbound }\end{array}$ & 23499 & -34 & 26821 & -32 \\
\hline $\begin{array}{l}\text { Across cordon } \\
\text { Outbound }\end{array}$ & 14858 & -32 & 15126 & -47 \\
\hline
\end{tabular}

\subsection{Optimizing toll locations and levels in the Stockholm network}

A total of six scenarios will be evaluated with respect to the social surplus. The first and second are the MSCP scheme and the current congestion pricing cordon in Stockholm (with the toll levels set to 20 SEK). In the third scenario, the toll locations are given by the current cordon but with the toll levels optimized. The fourth and fifth scenarios are based on the current cordon but with tolls (one in each direction) added on the bypass 
highway "Essingeleden" (marked in Figure 1); the fourth scenario with all toll levels being set to 20 SEK (denoted extended current cordon) and no optimization performed, and the fifth with all the toll levels optimized (denoted extended current cordon optimized). Finally, in the sixth scenario, (11) is solved to optimize both toll locations and toll levels (denoted TDP solution), using the smoothening technique described in Section 2.3.

When solving the TLP, the sensitivity analysis based ascent method has been initialized with toll levels equal to 20 SEK. For the smoothening technique, the initial value of $k$ is set to 1 , and the initial solution $\left(\tau^{0}\right)$ is set to zero tolls for all links. Note that the higher $k$ is in the first iteration, the fewer toll locations will be considered when all links are initially set to zero. On the other hand, a higher initial value on $k$ will reduce both the total number of iterations, and within each iteration fewer sensitivity computations have to be carried out, reducing the computational burden. Our experience is that $k=1$ and $\rho=0.25$, gives a reasonable balance between solution quality and computational time.

The range of the toll levels, the number of toll facilities, the number of links with flow about capacity and the total car demand, are for each scenario given in Table 3. In Table 4, the resulting consumer surplus, operator surplus and social surplus associated with each scenario are presented. $\Delta$ will imply a comparison with the no toll solution, and $\Delta S S^{0}$ will denote the change in social surplus if the set-up and operational costs are excluded. The maximum value on $\Delta S S^{0}$ can be obtained by maximizing (11) with $C_{a}=0$ for every link $a$ in $A$, which is one particular case for which (11) can be solved to global optimality by using MSCP tolls. In Figure 1, the current cordon, the bypass highway "Essingeleden" and the central business district (CBD) are displayed. 
Table 3: Toll levels $(\tau)$, number of toll collecting facilities $(N)$, number of links with flow exceeding capacity $(E)$ and total car demand $(q)$ in number of car trips per hour.

\begin{tabular}{lcccc}
\hline Scheme & $\tau$ (SEK) & $N$ & $E$ & $q$ \\
\hline O. No toll & 0 & 0 & 80 & 121946 \\
1. MSCP & $0.0-32.0$ & 291 & 21 & 102904 \\
2. Current cordon & 20 & 20 & 60 & 114215 \\
3. Current cordon optimized & $8.1-42.8$ & 20 & 54 & 111299 \\
4. Extd. current cordon & 20 & 22 & 54 & 112866 \\
5. Extd. current cordon optimized & $8.7-47.9$ & 22 & 45 & 108562 \\
6. TDP solution & $1.1-34.8$ & 69 & 24 & 105653 \\
\hline
\end{tabular}

Table 4: Consumer surplus ( $\triangle C S$ ), operator surplus ( $\triangle O S$ ), and social surplus with ( $\triangle S S$ and $\Delta S S^{0}$ ), in SEK for each scenario.

\begin{tabular}{lcccc}
\hline Scheme & $\Delta C S$ & $\Delta O S$ & $\Delta S S^{0}$ & $\Delta S S$ \\
\hline 1. MSCP & -1029351 & 1638650 & 1031496 & 609299 \\
2. Current cordon & -191037 & 494752 & 303715 & 273715 \\
3. Current cordon optimized & -39463 & 409299 & 399837 & 369837 \\
4. Extd. current cordon & -294881 & 690384 & 430503 & 395503 \\
5. Extd. current cordon optimized & -31790 & 580593 & 583803 & 548803 \\
6. TDP solution & -832404 & 1726488 & 993283 & 894083 \\
\hline
\end{tabular}

In the TDP solution the toll levels range between 1.1 and 34.8 SEK, and the demand is reduced by 16245 car journeys per hour from 121946 with the no toll solution. The number of links with a flow above model capacity is reduced from 80 to 24. With no tolls in the traffic network 35 links have flows which exceed capacity by more than $150 \%$, and with the TDP solution the flow on only three links exceeds the capacity with more than $125 \%$. This can be compared with the current closed cordon congestion scheme which has 60 links with flow above capacity, and 23 links with flow that exceeds $125 \%$ of the capacity.

In Table 4 it is clear that the maximum value of $\Delta S S^{0}$ is obtained with MSCP tolls. MSCP tolling (Scenario 1), however, require a large number of tolls to be located which reduce the value of $\Delta S S$. It can also be noted that none of the solutions based on the current closed cordon schemes come close to the $\Delta S S$ value of the TDP solution. 
Equal toll levels (Scenario 2 and 4) may still be appealing from a practical perspective. The MSCP scheme yields the second highest increase in social surplus, and comparing $\Delta S S^{0}$ for the different scenarios, optimizing toll location and levels results in $96 \%$ of the maximum $\Delta S S^{0}$, but with only 69 tolled links (24\% of the tollable links) compared with the 291 tolled links which are required to reach MSCP.

Examining the schemes based on the current cordon it is obvious that by tolling "Essingeleden", the current scheme can be improved, and with a toll of 20 SEK on every tolled link in the extended scheme, $\Delta S S$ is increased by $42 \%$, compared with the current cordon. We also notice that by re-optimizing the toll levels, instead of using the single one of 20 SEK, $\triangle S S$ can be improved between 35 and $39 \%$. The proportions between the consumer surplus (CS) and operator surplus (OS) for the two schemes based on the current Stockholm cordon (with and without a toll on "Essingeleden"), but with differentiated toll levels, is different from the other pricing schemes. The reoptimized schemes yield a reduced disutility for the road users, but the operator surplus is also reduced. It is, however, necessary to remember the non-convex nature of the problem, and the re-optimizations have been initialized with an equal toll level of 20 SEK for all tollable links. If zero toll levels would instead be used as initial toll levels in sensitivity based ascent method, the re-optimization of the current cordon (no toll on "Essingeleden") would result in $\Delta S S=366252$ SEK, but with $\triangle C S=-221391$ SEK and $\Delta O S=587644$ SEK. Thus, both the disutility for the road users and the operators surplus has increased compared with the current cordon. It is, however, no surprise that the final solution will depend on the starting point when using a steepest ascent method to solve a non-convex optimization problem. In this case it is interesting to see that even though the two solutions almost have the same objective function value, there are large differences in the disutility experienced by the road users, and the total sum of the 
collected tolls. If the operator is more interested of a low experienced disutility for the road users, the pricing scheme with $\triangle C S=-39463$ SEK is preferable, but if the operator prefers a high profit $\Delta C S=587644$ SEK is the one to choose.

The changes in car travel times for four different OD pairs have been studied more carefully, comparing the TDP solution, the current cordon (equal tolls, 20 SEK) and the no tolled solution. One OD pair with origin within the $\mathrm{CBD}$, defined by the current closed cordon in Stockholm (see Figure 1) and destination in the southern suburbs (a), the second one originating in the southern suburbs and ending within the CBD (b), the third one with both origin and destination within the CBD (c), and the fourth with origin in the northern and destination in the southern suburbs (d). Table 5 displays the maximal and the minimal travel times, the maximal and the minimal paid tolls, and the total travel costs for the four OD pairs and the three scenarios.

For travellers in OD pair (a) and (c) the reductions in travel time is small, and for OD pair (c) the reduction do not differ very much between the TDP solution and the current cordon, even though the users traveling within the current cordon today do not need to pay any tolls. Also, for OD pair (a) and (c) the reduction in travel time is larger with the current cordon, compared to the TDP solution. In the TDP solution OD pair (c) gets a reduction in travel time that is larger than the time equivalent to the paid tolls. For the current closed cordon scheme we notice that no tolls have to be paid for journeys within OD pair (c) and (d), which is not surprising since (c) is a journey within the current cordon and road users in OD pair (d) do not need to pass the current cordon to reach their destination. Focusing on the TDP solution the large reductions in travel time can be found for road users in OD pair (b) and (d), and these are also the journeys with the highest paid tolls. 
Table 5: Travel time, paid tolls and total cost for a car journey in four different OD pairs.

\begin{tabular}{ccccc}
\hline OD pair & Scheme & $\begin{array}{c}\text { Travel time } \\
\text { (minutes/traveller) }\end{array}$ & $\begin{array}{c}\text { Paid tolls } \\
\text { (SEK/car) }\end{array}$ & $\begin{array}{c}\text { Total cost } \\
\text { (SEK/traveller) }\end{array}$ \\
\hline \multirow{4}{*}{$a$} & TDP solution & $37.0-37.3$ & $13.6-14.0$ & 56.8 \\
& Current cordon & 36.5 & 20.0 & 61.5 \\
No toll & 40.0 & - & 48.0 \\
\hline \multirow{4}{*}{ b } & TDP solution & $44.3-45.0$ & $47.6-48.5$ & 95.3 \\
& Current cordon & 60.6 & 20.0 & 90.4 \\
& No toll & 66.1 & - & 79.3 \\
\hline \multirow{4}{*}{$d$} & TDP solution & 29.7 & 2.9 & 38.2 \\
& Current cordon & 28.7 & - & 34.4 \\
& No toll & 31.9 & - & 38.3 \\
\hline & TDP solution & 66.5 & 50.4 & 124.4 \\
& Current cordon & 82.9 & - & 99.5 \\
& No toll & 84.2 & - & 101.0 \\
\hline
\end{tabular}




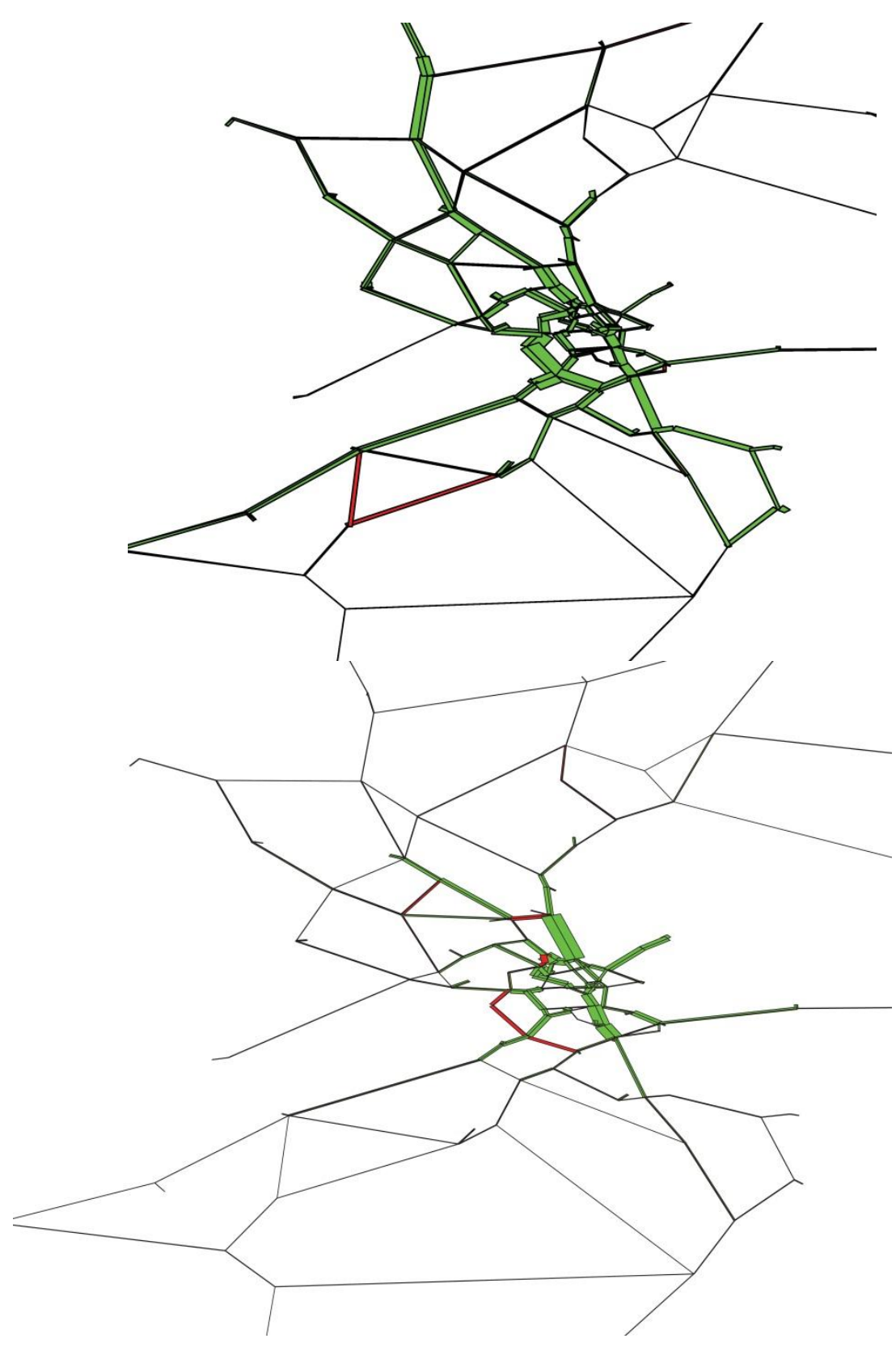

Figure 2: Link flow changes with the TDP solution (upper) and with the current closed cordon (lower) compared to the no toll solution. Reduced flow is marked with green and increased with red.

The link flows in the TDP and in the current cordon solutions have been compared to the link flows in the no toll solution. Figure 2 shows a link flow comparison for the major part of the Stockholm network. Green bars indicate a reduction in link flow and red bars an increase. The TDP solution gives a reduction of the traffic flow, not only 
within the CBD but also in the surrounding network, while the current cordon only has a larger impact on the traffic flow in and out of the CBD. Table 6 gives the change in traffic flow across the current congestion pricing cordon in Stockholm. The TDP and MSCP solutions have similar effect on the flow crossing the cordon in both directions and the change in the inbound flow from these solutions are also close to what is achieved with the current cordon. For the outbound flow, both the TDP and MSCP solutions give less reduction. The optimized versions of the current cordon (both with and without a toll on "Essingeleden") shows a larger change in the inbound traffic flow, and smaller change in the outbound traffic flow, compared with the current cordon. As the current cordon in Stockholm is constructed, it is likely that the traffic flow on "Essingeleden" will increase and create severe congestion. In Table 7 the volume/capacity (V/C) ratio, from each scenario, is given for "Essingeleden". Both the current cordon and its re-optimized version have small effects on the northbound traffic on "Essingeleden", which is the direction with the most sever congestion. For the southbound direction there is an increase by $14-17 \%$. The extended cordon decreases the $\mathrm{V} / \mathrm{C}$ ratio between 12 and $19 \%$, and with the toll levels optimized, this cordon has a major impact with a reduction around $40 \%$ in both directions. The TDP and MSCP schemes show similar V/C ratios, with reductions between 23 and $26 \%$ in the southbound direction and around $32 \%$ in the northbound direction. Over all the TDP solution increases the flow on very few links and none of the links with increased flow exceeds the link capacity. 
Table 6: Change in traffic flow across the current cordon in Stockholm compared with the no toll solution.

\begin{tabular}{lccc}
\hline Scheme & Total & Inbound & Outbound \\
\hline 1. MSCP & $-23 \%$ & $-28 \%$ & $-16 \%$ \\
2. Current cordon & $-37 \%$ & $-32 \%$ & $-47 \%$ \\
3. Current cordon optimized & $-47 \%$ & $-49 \%$ & $-42 \%$ \\
4. Extd. current cordon & $-36 \%$ & $-31 \%$ & $-46 \%$ \\
5. Extd. current cordon optimized & $-42 \%$ & $-45 \%$ & $-37 \%$ \\
6. TDP solution & $-23 \%$ & $-30 \%$ & $-12 \%$ \\
\hline
\end{tabular}

Table 7: Volume/capacity (V/C) ratio for "Essingeleden", change (in \%) compared with the no toll solution.

\begin{tabular}{lcc}
\hline Scheme & Southbound & Northbound \\
\hline O. No toll & 0.65 & 1.02 \\
1. MSCP & $0.50(-23 \%)$ & $0.70(-31 \%)$ \\
2. Current cordon & $0.74(+14 \%)$ & $1.03(+1 \%)$ \\
3. Current cordon optimized & $0.76(+17 \%)$ & $1.03(+1 \%)$ \\
4. Extd. current cordon & $0.57(-12 \%)$ & $0.83(-19 \%)$ \\
5. Extd. current cordon optimized & $0.39(-40 \%)$ & $0.56(-45 \%)$ \\
6. TDP solution & $0.48(-26 \%)$ & $0.68(-33 \%)$ \\
\hline
\end{tabular}

In practice, fractional toll levels are not likely to be introduced. Given a toll level solution to either (10) or (11), the continuous toll levels are therefore replaced by the closest multiple of 5 SEK. This is done for one discrete toll level at a time, starting with the links with tolls closest to the lowest discrete toll level, and in between the remaining non-fixed toll levels are re-optimized. Figure 3 shows the toll locations and the discretizised toll levels based on the TDP solution. For the discrete toll levels, $\triangle S S$ is 871062 SEK which is $97 \%$ of that with the original TDP solution. The toll levels are 5, 10, 15, 20, 25 and 35 SEK, since no toll came close to 30 SEK. In the process one toll is set to zero (with an initial toll level below 2.5 SEK). As a comparison we round all toll levels given by the TDP solution to the closest discrete level (multiples of 5 SEK), without any re-optimization in between, which only reduce $\Delta S S$ by another 5542 SEK. Thus the benefit from re-optimizing the toll levels during the discretization process, for this case, is neglectable. 


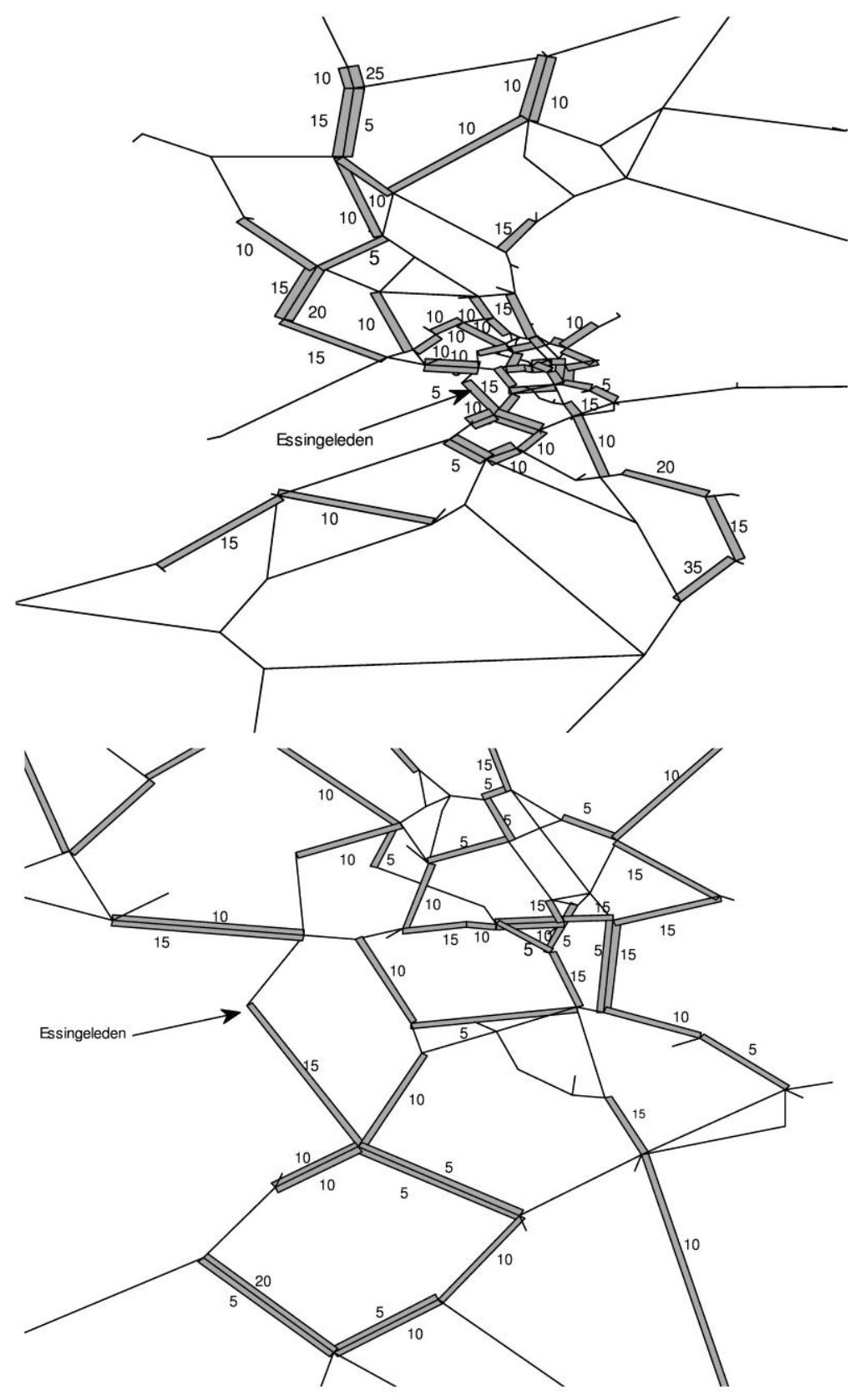

Figure 3: The complete set of toll locations and discrete levels given by the discretizised solution. The upper network shows the major part of the Stockholm network, and the lower one is a detailed view of the central part.

\subsection{Optimal expansion of the current congestion pricing scheme in Stockholm}

How to extend an existing congestion pricing scheme is an interesting question. Since the toll collecting facilities are already in place, it is both practical and economical to make use of the existing facilities when planning an extension of the current congestion 
pricing scheme. This is reasonable also from the travellers' perspective, as they are already used to the current system. The smoothening technique can easily be adopted to solve this problem, by fixing the toll locations of the current toll collecting facilities.

When considering an optimal expansion of the current congestion pricing cordon in Stockholm, the current locations (Figure 1) and toll levels (20 SEK) are given as fixed and the question is where to locate additional tolls. It is further assumed that it is only practical to toll the larger arterial roads. This leaves a total of 174 possible toll locations. Applying the smoothening technique result in 40 new toll locations in addition to the 20 current locations. For the additional toll locations the toll levels range from 3.0 to $25.4 \mathrm{SEK}$, with $\Delta S S=822905$, only including the setup and operational costs of the additional toll locations, with $\Delta S S^{0}=887405$ which makes up $86 \%$ of the maximum $\Delta S S^{0}$. The same procedure for finding discrete toll levels based on the continuous ones, as discussed in the previous section, has also been applied to the expansion. The additional toll locations and discrete toll levels are presented in Figure 4, and the discrete toll levels yields $98.4 \%$ of $\Delta S S$ with continuous ones. 


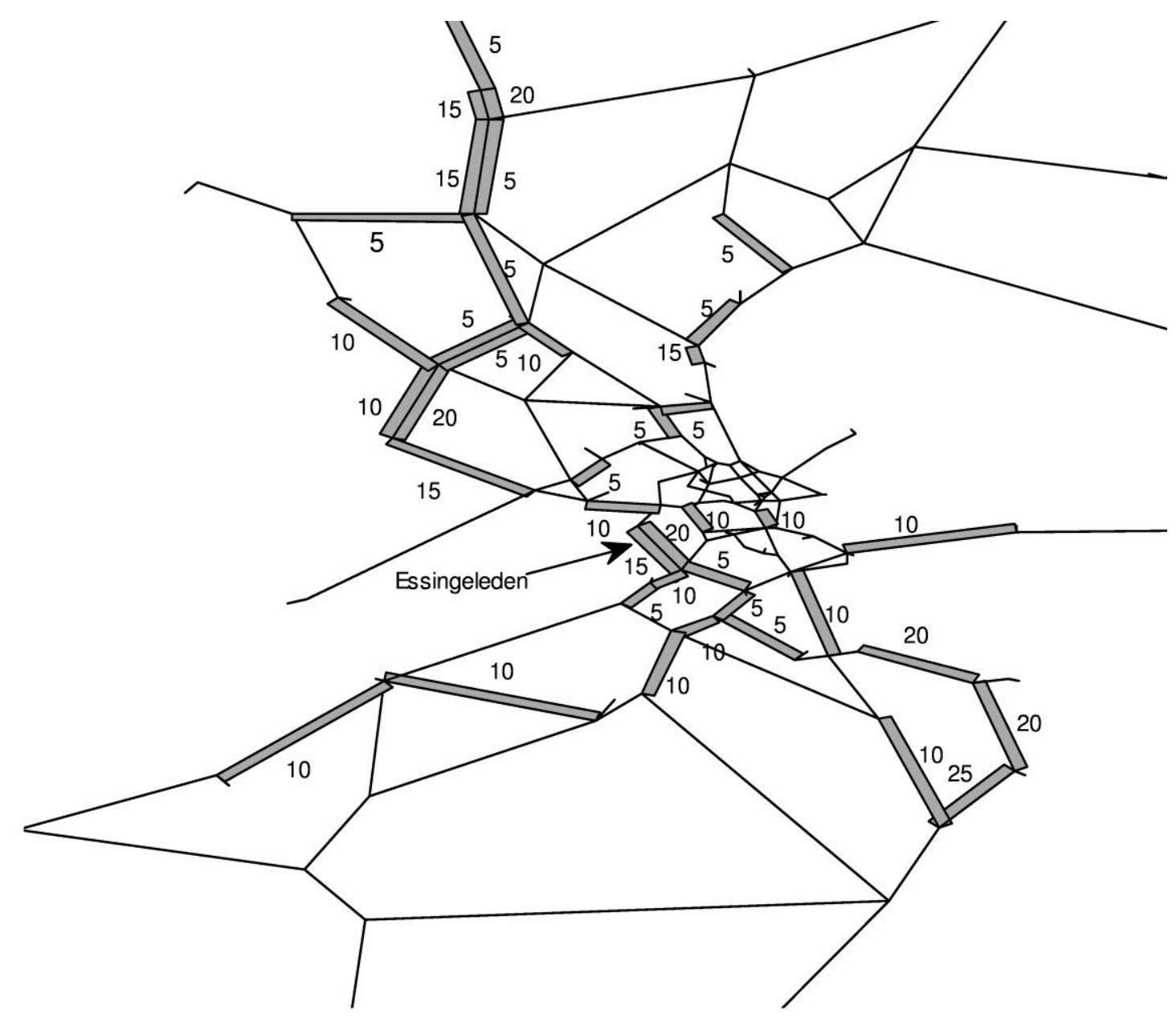

Figure 4: Additional toll locations and discrete toll levels in multiples of 5 SEK for the expanded pricing scheme.

\section{Conclusions and further research}

The case study presented in this paper shows that the smoothening technique, used for solving the TDP, is applicable for networks of natural size. It is further shown that it is possible to achieve $96 \%$ of the maximum social surplus (disregarding any setup or operational cost) by only locating a toll on $24 \%$ of the tollable links, which significantly reduce the size and cost of the toll collection system, compared with the MSCP solution. The results also provide insight into benefits that can be achieved by optimization of the toll levels in the current cordon in Stockholm, and/or adding tolls on "Essingeleden". Allowing differentiated toll levels opens for individual optimization of 
the level at each toll point, and just by optimizing the toll levels in the current cordon the social surplus can be increased by $35 \%$, without any new toll locations being added. It has also been shown that even though two different local optimal solutions to the TLP (found with different initial solutions) have similar objective function values, they may differ significantly when it comes to the level of consumer and operator surpluses.

The Wardrop equilibrium assumes that the road users have perfect information about the travel cost in the road network. Allowing continuous toll levels may, however, make it more difficult for the road users to overview the cost of making a trip in the network. From this perspective discrete toll levels are more appealing in practice. The introduction of discrete toll levels would, however, make any kind of derivative based algorithm useless in the optimization process. For the case presented in this paper it has been shown how continuous toll levels easily can be replaced by discrete ones, with only a small reduction of the social surplus. These results of course depend on the number of discrete toll levels, but for the Stockholm network the use of six discrete toll levels only reduce the improvement in social surplus by $3 \%$.

There can be several reasons to keep the existing toll collection facilities, and the heuristic approach for locating the tolls can be applied to find additional toll locations. For the aggregated Stockholm network it is possible to reach $86 \%$ of the maximum social surplus, with 40 additional toll locations.

In this study, only the morning rush hour is studied. Evaluation and optimization of the toll locations given the different demand pattern during different time periods is an interesting area for further research. Alternative functional form for approximating the discrete variables may further improve the results. When it comes to large networks the computational burden will be large, and to find incremental schemes of the $k$ 
parameter, possibly individual for each link, could both improve the results and cut the computational time, by reducing the number of overall iterations.

\section{Acknowledgements}

The authors would like to thank Torbjörn Larsson for comments on an earlier draft of this paper.

\section{References}

Algers, S., A. Daly, P. Kjellman, and S. Widlert. 1996. “'Stockholm model system SIMS: Application." In World Transport Research. Proceedings of the 7th World Conference on Transport Research, edited by Hensher, D. A., J. King, and T. H. Oum: 345-361. Oxford: Elsevier.

Beckmann, M., C. McGuire, and C. B. Winsten. 1956. Studies in the Economics of Transportation. New Haven: Yale University Press.

Beser, M., and S. Algers. 2002. 'SAMPERS, The New Swedish National Travel Demand Forecasting Tool." In National Transport Models: Recent Developments and Prospects, Advances in Spatial Science, edited by Lundkvist, L., and L. G. Mattsson: 101-118. Berlin: Springer Verlag.

Ekström, Joakim. (2008). “Designing Urban Road Congestion Charging Systems: Models and Heuristic Solution Approaches" Licentiate Thesis, Linköping University.

Ekström, J., L. Engelson, and C. Rydergren. 2009. “Heuristic algorithms for a secondbest congestion pricing problem." NETNOMICS: Economic Research and Electronic Networking 10 (1): 85-102.

Eliasson, J. 2009. “'A cost-benefit analysis of the Stockholm congestion charging system."' Transportation Research Part A: Policy and Practice 43 (4): 468-480. 
Eliasson, J., L. Hultkrantz, L. Nerhagen, and L. S. Rosqvist. 2009. “'The Stockholm congestion - charging trial 2006: Overview of effects." Transportation Research Part A: Policy and Practice 43 (3): 240-250.

Engelson, L., and S. Svalgård. 1995. “The travel demand and land-use model for Stockholm region."' Technical report, Inregia AB.

Evans, S. P. 1976. ' Derivation and analysis of some models for combining trip distribution and assignment."' Transportation Research 10 (1): 37-57.

Josefsson, M. and M. Patriksson. 2007. “'Sensitivity analysis of separable traffic equilibrium equilibria with application to bilevel optimization in network design." Transportation Research Part B: Methodological 41 (1): 4-31.

Karlström, A. and J. P. Franklin. 2009. “'Behavioral adjustments and equity effects of congestion pricing: Analysis of morning commutes during the Stockholm trial." Transportation Research Part A: Policy and Practice 43 (3): 283-296.

Knight, F. 1924. "Some fallacies in the interpretation of social cost." Quarterly Journal of Economics 38 (4): 582-606.

Kumar, A. 1980. "Pivot point modeling procedures in demand estimation." Transportation Engineering Journal 106 (6): 647-660.

Larsson, T. and M. Patriksson. 1992. 'Simplicial decomposition with disaggregated representation for the traffic assignment problem."' Transportation Science 26 (1): 4-17.

Larsson, T. and M. Patriksson. 1998. 'Side constrained traffic equilibrium modelstraffic management through link tolls." In Equilibrium and advanced transportation modeling, edited by P. Marcotte and S. Nguyen, 125-151. Norwell: Kluwer Academic.

Lawphongpanich, S. and D. W. Hearn 2004. “An MPEC approach to second-best toll pricing." Mathematical Programming 101 (1): 33-55. 
Lederman, R. and L. Wynter (2011). “'Real-time traffic estimation using data expansion." Transportation Research Part B: Methodological 45 (7): 1062-1079.

Liu, L. N. and J. F. McDonald. 1999. “Economic efficiency of second-best congestion pricing schemes in urban highway systems." Transportation Research Part B: Methodological 33 (3): 157-188.

Lundgren, J. T. and A. Peterson. 2008. "A heuristic for the bilevel origin-destinationmatrix estimation problem." Transportation Research Part B: Methodological 42 (4): $339-354$

Luo, Z., J. Pang, and D. Ralph. 1996. Mathematical programs with equilibrium constraints. Cambridge: Cambridge University Press.

Migdalas, A. 1995. 'Bilevel programming in traffic planning: Models, methods and challenge." Journal of Global Optimization 7 (4): 381-405.

Patriksson, M. 1994. The Traffic Assignment Problem: Models and Methods. Utrecht: VSP.

Patriksson, M. and R. T. Rockafellar. 2003. 'Sensitivity analysis of aggregated variational inequality problems, with application to traffic equilibria." Transportation Science 37 (1): 56-68.

Pigou, A. C. 1920. Welth and Welfare. London: MacMillan.

Sheffi, Y. 1985. Urban Transportation Networks: Equilibrium Analysis with Mathematical Programming Methods. New Jersey: Prentice-Hall.

Shepherd, S. and A. Sumalee. 2004. “'A genetic algorithm based approach to optimal toll level and location problems". Networks and Spatial Economics 4 (2): 161-179. 
SIKA. 2009. Värden och metoder för transportl-sektorns samhälls\-ekonomiska analyser - ASEK 4. SIKA Rapport 2009:3. SIKA - Statens institut för kommunikationsanalys.

Sumalee, A. 2004. “Optimal road user charging cordon design: A heuristic optimization approach." Computer-Aided Civil and Infrastructure Engineering 19(5): 377-392.

Transek. 2003. Miljöavgifter i Stockholm, analys av nuvarande förslag till utformning. Technical report, Transek AB.

Transek. 2004. Miljöavgifter i Stockholm, effekter av kommunstyrelsens förslag till utformning december 2003. Technical report, Transek AB.

Verhoef, E. T. 2002. 'Second-best congestion pricing in general static transportation networks with elastic demands." Regional Science and Urban Economics 32 (3): 281310.

Verhoef, E. T., P. Nijkamp, and P. Rietveld. 1996. 'Second-best congestion pricing: the case of an untolled alternative." Journal of Urban Economics 40 (3): 279-302.

Wardrop, J. 1952." Some theoretical aspects of road traffic research."' In Proceedings of the Institute of Civil Engineers 1 (2): 325-378.

Yang, H. and W. H. K. Lam. 1996. “'Optimal road tolls under conditions of queueing and congestion." Transportation Research Part A: Policy and Practice 30 (5): 319-332.

Yang, H. and X. Zhang (2003). “Optimal toll design in second-best link-based congestion pricing." Transportation Research Records 1857: 85-92.

Yildirim, B. and D. W. Hearn. 2005. “A first best toll pricing framework for variable demand traffic assignment problems." Transportation Research Part B: Methodological 39 (8): 659-678. 
Yin, Y. and S. Lawphongpanich 2009. “'Alternative marginal-cost pricing for road networks." NETNOMICS: Economic Research and Electronic Networking 10 (1): $77-$ 83.

Zerbe, R. O. J. and D. D. Dively. 1994. Benefit-cost analysis in theory and practice. New York: HarperCollins.

Zhang, X. and H. Yang (2004). “The optimal cordon-based network congestion pricing problem." Transportation Research Part B: Methodological 38 (6): 517-537. 